\title{
Type II balanced truncation for deterministic bilinear control systems
}

\author{
Martin Redmann*
}

June 20, 2018

\begin{abstract}
When solving partial differential equations numerically, usually a high order spatial discretisation is needed. Model order reduction (MOR) techniques are often used to reduce the order of spatially-discretised systems and hence reduce computational complexity. A particular MOR technique to obtain a reduced order model (ROM) is balanced truncation (BT), a method which has been extensively studied for deterministic linear systems. As so-called type I BT it has already been extended to bilinear equations, an important subclass of nonlinear systems. We provide an alternative generalisation of the linear setting to bilinear systems which is called type II BT. The Gramians that we propose in this context contain information about the control. It turns out that the new approach delivers energy bounds which are not just valid in a small neighbourhood of zero. Furthermore, we provide an $\mathcal{H}_{\infty}$-error bound which so far is not known when applying type I BT to bilinear systems.
\end{abstract}

Keywords: Model order reduction, balanced truncation, bilinear systems, Gramians, error bound.

MSC classification: 93A15, 93B05, 93B07, 93B36, 93C10.

\section{Introduction}

Numerical simulations are one of the conventional methods to study physical phenomena of dynamical systems. However, extracting all the complex system dynamics generally leads to large state-space systems, whose direct simulations are inefficient and involve huge computational cost. Hence, there is a need to consider model order reduction (MOR), aiming at replacing these large-scale systems by systems of much smaller state dimension. MOR for linear systems has been investigated intensively in recent years and is widely used in numerous applications, see e.g. [1, 7, 26]. In this work, we consider MOR for bilinear control systems, which can be considered as a bridge between linear

\footnotetext{
${ }^{*}$ Weierstrass Institute for Applied Analysis and Stochastics, Mohrenstr. 39, 10117 Berlin, Germany, Email: martin.redmann@wias-berlin.de. Financial support by the DFG via Research Unit FOR 2402 is gratefully acknowledged.
} 
and nonlinear systems. Applications of bilinear systems can be seen in various fields [10, 19, 24].

Several methods for linear systems have been extended to bilinear systems such as balanced truncation (BT) [4] or other balancing related methods [17. Moreover, interpolation-based MOR has been applied [2, 3, 9, 13. In this manuscript, we focus on BT for bilinear systems which for linear systems has been studied in e.g. [1, 20]. Later on, the balancing concept for general nonlinear systems has been extended in a series of papers, see e.g. [14, 16, 25].

BT relies on controllability/reachability and observability Gramians. In [4] Gramians were proposed which we will call type I (bilinear) Gramians in this paper. The drawback of this approach is that only local energy bounds are available [15]. Furthermore, no error bound has been proved so far. The type I bilinear Gramians play a role for stochastic systems as well [4, 8, 23], where they are also used in the context of balancing. Recently, a second way of balancing for stochastic systems was discussed [5, 12, 21, 22]. It is based on another reachability Gramian and called type II ansatz. With this approach an $\mathcal{H}_{\infty}$-error bound can be achieved which cannot be proved in the ansatz used in [8, 23].

In this paper, we introduce type II bilinear Gramians in Section 2 which are inspired by the type II stochastic Gramians. The difference lies in additional information about the control in the bilinear Gramians. Under the assumption of having bounded controls, we then prove bounds for the reachability and observability energy of the underlying bilinear system using the type II bilinear Gramians. This provides a motivation to balance the bilinear system based on the new Gramians. The procedure will be explained in Section 3. In Section 4, an $\mathcal{H}_{\infty}$-error bound for type II bilinear BT will be proved, again assuming bounded controls. This error bound is the main result of this paper and has the same structure as in the linear case. Error bounds for BT applied to bilinear system did not exist before.

\section{Setting and type II Gramians}

We consider the following bilinear deterministic equation

$$
\frac{d x(t)}{d t}=A x(t)+B u(t)+\sum_{i=1}^{m} N_{i} x(t) u_{i}(t), \quad x(0)=x_{0}, \quad t \geq 0,
$$

where $A \in \mathbb{R}^{n \times n}, B \in \mathbb{R}^{n \times m}$ and $N_{1}, \ldots, N_{m} \in \mathbb{R}^{n \times n}$. Below, $x\left(t, x_{0}, u\right), t \geq 0$, denotes the solution to (11) with initial condition $x_{0} \in \mathbb{R}^{n}$ and control process $u=\left(u_{1}, \ldots, u_{m}\right)^{T}$. In our framework the state equation (1) is additionally equipped with an output equation

$$
y\left(t, x_{0}, u\right)=C x\left(t, x_{0}, u\right), \quad t \geq 0,
$$

where $C \in \mathbb{R}^{p \times n}$. The control $u$ is usually assumed to be an $L_{T}^{2}$ function meaning that

$$
\|u\|_{L_{T}^{2}}^{2}:=\int_{0}^{T} u^{T}(t) u(t) d t=\int_{0}^{T}\|u(t)\|_{2}^{2} d t<\infty
$$


for every $T>0$. Moreover, a classical assumption is the asymptotic stability of the uncontrolled equation (1), that is

$$
\left\|x\left(t, x_{0}, 0\right)\right\|_{2} \rightarrow 0
$$

for $t \rightarrow \infty$ and every $x_{0} \in \mathbb{R}^{n}$, being equivalent to the Hurwitz property of $A$. Later on we will introduce a further condition on $u$ and a stronger assumption on the stability. This is required to define the type II Gramians.

Before alternative Gramians are discussed, the existing theory will be summarised below.

Type I reachability and observability Gramians In [4 and [15 bounds for the controllability/reachability and observability energy of the equations (11) and (2) have been proved using certain Gramians. We call these matrices type I Gramians here. The type I reachability Gramian $P_{1}$ is the unique solution to

$$
A P_{1}+P_{1} A^{T}+\sum_{i=1}^{m} N_{i} P_{1} N_{i}^{T}=-B B^{T},
$$

whereas the type I observability Gramian is defined as the unique solution of

$$
A^{T} Q_{1}+Q_{1} A+\sum_{i=1}^{m} N_{i}^{T} Q_{1} N_{i}=-C^{T} C .
$$

Equations (4) and (5) are also considered in the context of model order reduction for stochastic systems [4, 8]. A further discussion about these Gramians can be found in [21. A unique positive semidefinite solution to the identities (44) and (5) exists if the following stability condition holds:

$$
\sigma\left(I_{n} \otimes A+A \otimes I_{n}+\sum_{i=1}^{m} N_{i} \otimes N_{i}\right) \subset \mathbb{C}_{-} .
$$

Property (6) is also called asymptotic mean square stability because it represents a stability concept for stochastic systems [8, 11, 18, 21]. It is satisfied if $A$ is asymptotically stable and the matrices $N_{i}$ are relatively small (in some norm) compared to the eigenvalues of $A$. That is why it is actually enough to assume (3) because equation (11) can be rescaled as

$$
\frac{d x(t)}{d t}=A x(t)+\left[\frac{1}{\gamma} B\right][\gamma u(t)]+\sum_{i=1}^{m}\left[\frac{1}{\gamma} N_{i}\right] x(t)\left[\gamma u_{i}(t)\right]
$$

where the weighted matrices $\tilde{N}_{i}=\frac{1}{\gamma} N_{i}$ can be made arbitrary small with a sufficiently large constant $\gamma>0$. 
No let us introduce the energy functionals. As in [4, 15, 25] the controllability energy is

$$
E_{c}\left(x_{0}\right):=\min _{\substack{\left.\left.u \in L^{2}(]-\infty, 0\right]\right) \\ x\left(-\infty, x_{0}, u\right)=0}} \int_{-\infty}^{0}\|u(t)\|_{2}^{2} d t .
$$

In [4] and [15] the observability energy is considered for the case $B=0$, whereas for general non-linear systems $u \equiv 0$ is assumed [25]. With $B=0$, the observability energy is

$$
E_{o}\left(x_{0}\right):=\max _{\substack{u \in L^{2}([0, \infty]) \\\|u\|_{L^{2}}<\alpha, B=0}} \int_{0}^{\infty}\left\|y\left(t, x_{0}, u\right)\right\|_{2}^{2} d t, \text { for } \alpha>0 \text { small. }
$$

The next theorem is a result from [4] and [15].

Theorem 2.1. Let $A$ be asymptotically stable (and at least one $N_{i}$ of full rank). If there are positive definite solutions $P_{1}$ and $Q_{1}$ to (4) and (5), then there exists a neighbourhood $\hat{W}(0)$ of zero such that

$$
x_{0}^{T} P_{1}^{-1} x_{0} \leq E_{c}(x) \quad \text { and } \quad E_{o}(x) \leq x_{0}^{T} Q_{1} x_{0} \quad \text { for } x \in \hat{W}(0) .
$$

The above inequalities allow us to identify the hardly controllable and observable states (at least locally). Those are contained in the eigenspaces spanned by the eigenvectors of $P_{1}$ and $Q_{1}$, respectively, that correspond to the small eigenvalues.

More accurate bounds than in Theorem 2.1] were obtained for truncated Gramians [6], which are also computationally cheaper than the type I Gramians.

Type II reachability and observability Gramians We now introduce alternative Gramians which we will see, guarantee an error bound for the bilinear system. The so called type II Gramians are inspired by the stochastic case. A postive definite reachability Gramian $P_{2}$ which solves

$$
A^{T} P_{2}^{-1}+P_{2}^{-1} A+\sum_{i=1}^{m} N_{i}^{T} P_{2}^{-1} N_{i} \leq-P_{2}^{-1} B B^{T} P_{2}^{-1}
$$

was initially introduced in [12] in order to show the existence of an $\mathcal{H}_{\infty}$-error bound for BT applied to stochastic systems. There the balancing was based on $P_{2}$ and the type I Gramian $Q_{1}$, the solution to (5). The stochastic Gramian $P_{2}$ was furthermore analysed in [5] and used in [21, 22] in a more general form.

An inequality is considered in (8) since the existence of a positive definite solution is not ensured when having an equality. A solution to inequality (8) exists if condition (6) is satisfied [12, 21]. As already mentioned above, (6) can be weakened to the assumption of asymptotic stability of $A$ since $N_{i}$ can be made arbitrary small.

We will not take the matrices $Q_{1}$ and $P_{2}$ (stochastic type II balancing) to introduce a type II approach for bilinear systems. Moreover, we will modify them further. The 
idea is to let information about the control enter the new Gramians. This is done by choosing a constant $k>0$. So, the type II Gramians of the bilinear system (11), (2) are given by a perturbed matrix $A$ :

$$
\begin{aligned}
\left(A+\frac{k^{2}}{2} I_{n}\right)^{T} P^{-1}+P^{-1}\left(A+\frac{k^{2}}{2} I_{n}\right)+\sum_{i=1}^{m} N_{i}^{T} P^{-1} N_{i} & \leq-P^{-1} B B^{T} P^{-1}, \\
\left(A+\frac{k^{2}}{2} I_{n}\right)^{T} Q+Q\left(A+\frac{k^{2}}{2} I_{n}\right)+\sum_{i=1}^{m} N_{i}^{T} Q N_{i} & =-C^{T} C .
\end{aligned}
$$

At the same time we suppose to have a control which is uniformly bounded by the perturbing constant on a finite time interval $[0, T]$

$$
\|u(t)\|_{2} \leq k, \quad t \in[0, T] .
$$

Of course $k$ cannot be arbitrary large because we need the existence of the solutions to (9) and (10). Ideally $k$ is such that

$$
\sigma\left(I_{n} \otimes\left(A+\frac{k^{2}}{2} I_{n}\right)+\left(A+\frac{k^{2}}{2} I_{n}\right) \otimes I_{n}+\sum_{i=1}^{m} N_{i} \otimes N_{i}\right) \subset \mathbb{C}_{-}
$$

holds. We observe that the type II Gramians $P, Q$ are coupled with the control $u$. We allow large controls $\left(L^{\infty}\right.$-sense) if the system is relatively stable (largest real part of the eigenvalues of $A$ is small and $N_{i}$ are small) and only small controls are admissible if the system is close to be unstable. We can again weaken condition (12) and only assume $\sigma\left(A+\frac{k^{2}}{2}\right) \subset \mathbb{C}_{-}$since we can rescale $N_{i}$, i.e., we replace it by $\tilde{N}_{i}=\frac{1}{\gamma} N_{i}$. In this situation we pay a price for the rescaling since if we want to bound the rescaled control $\tilde{u}=\gamma u$ as in (11), it is required to have $\|u(t)\|_{2} \leq \frac{k}{\gamma}$ which restricts the controls even more. From now on, we assume that (11) and (12) hold at the same time, knowing that we need a less restrictive assumption than (12) when using a smaller bound for the controls.

Let us now investigate how much energy is necessary if we control the system from zero into a certain direction. We desire to bound the reachability energy with the type II Gramian $P$. Let $\left(p_{k}\right)_{k=1, \ldots, n}$ be eigenvectors of $P$ such that they represent an orthonormal basis of $\mathbb{R}^{n}$. The corresponding eigenvalues are denoted by $\left(\lambda_{k}\right)_{k=1, \ldots, n}$. Then, for $t \in[0, T]$

$$
\begin{aligned}
\left\langle x(t, 0, u), p_{k}\right\rangle_{2}^{2} & \leq \lambda_{k} \sum_{i=1}^{n} \lambda_{i}^{-1}\left\langle x(t, 0, u), p_{i}\right\rangle_{2}^{2}=\lambda_{k}\left\|\sum_{i=1}^{n} \lambda_{i}^{-\frac{1}{2}}\left\langle x(t, 0, u), p_{i}\right\rangle_{2} p_{i}\right\|_{2}^{2} \\
& =\lambda_{k}\left\|P^{-\frac{1}{2}} x(t, 0, u)\right\|_{2}^{2}=\lambda_{k}\left[x(t, 0, u)^{T} P^{-1} x(t, 0, u)\right] .
\end{aligned}
$$


To shorten the notation we write $x(t)$ instead of $x(t, 0, u)$ from time to time below. So, by the product rule and by inserting (1), we have

$$
\begin{aligned}
x^{T}(t) P^{-1} x(t)= & \int_{0}^{t} x^{T}(s) P^{-1} d x(s)+\int_{0}^{t} d x^{T}(s) P^{-1} x(s) \\
= & \int_{0}^{t} x^{T}(s) P^{-1} A x(s) d s+\int_{0}^{t} x^{T}(s) P^{-1} B u(s) d s \\
& +\int_{0}^{t} x^{T}(s) A^{T} P^{-1} x(s) d s+\int_{0}^{t} u^{T}(s) B^{T} P^{-1} x(s) d s \\
& +\sum_{i=1}^{m}\left(\int_{0}^{t} x^{T}(s) P^{-1} N_{i} x(s) u_{i}(s) d s+\int_{0}^{t} x^{T}(s) u_{i}(s) N_{i}^{T} P^{-1} x(s) d s\right) .
\end{aligned}
$$

We analyse the last term above which can be written as

$$
\begin{aligned}
& \sum_{i=1}^{m} 2 \int_{0}^{t} x^{T}(s) P^{-1} N_{i} x(s) u_{i}(s) d s=\sum_{i=1}^{m} 2 \int_{0}^{t}\left\langle P^{-\frac{1}{2}} x(s) u_{i}(s), P^{-\frac{1}{2}} N_{i} x(s)\right\rangle_{2} d s \\
& \leq \sum_{i=1}^{m}\left(\int_{0}^{t}\left\|P^{-\frac{1}{2}} x(s) u_{i}(s)\right\|_{2}^{2} d s+\int_{0}^{t}\left\|P^{-\frac{1}{2}} N_{i} x(s)\right\|_{2}^{2} d s\right) \\
& =\int_{0}^{t} x^{T}(s) P^{-1} x(s)\|u(s)\|_{2}^{2} d s+\sum_{i=1}^{m} \int_{0}^{t} x^{T}(s) N_{i}^{T} P^{-1} N_{i} x(s) d s \\
& \leq \int_{0}^{t} x^{T}(s) P^{-1} k^{2} x(s) d s+\sum_{i=1}^{m} \int_{0}^{t} x^{T}(s) N_{i}^{T} P^{-1} N_{i} x(s) d s
\end{aligned}
$$

using the bound in (11). Summarising the above steps, we obtain

$$
\begin{gathered}
\left\langle x(t, 0, u), p_{k}\right\rangle_{2}^{2} \leq \lambda_{k}\left[\int_{0}^{t} x^{T}(s)\left(A^{T} P^{-1}+P^{-1} A+\sum_{i=1}^{m} N_{i}^{T} P^{-1} N_{i}+k^{2} P^{-1}\right) x(s) d s\right. \\
\left.+2 \int_{0}^{t} x^{T}(s) P^{-1} B u(s) d s\right] .
\end{gathered}
$$

Plugging in (9) yields

$$
\begin{aligned}
\left\langle x(t, 0, u), p_{k}\right\rangle_{2}^{2} & \leq \lambda_{k} \int_{0}^{t} 2 x^{T}(s) P^{-1} B u(s)-x^{T}(s) P^{-1} B B^{T} P^{-1} x(s) d s \\
& =\lambda_{k} \int_{0}^{t}\|u(s)\|_{2}^{2} d s-\left\|B^{T} P^{-1} x(s)-u(s)\right\|_{2}^{2} d s .
\end{aligned}
$$

Consequently, we have

$$
\lambda_{k}^{-\frac{1}{2}} \sup _{t \in[0, T]}\left|\left\langle x(t, 0, u), p_{k}\right\rangle_{2}\right| \leq\|u\|_{L_{T}^{2}} .
$$


So, by (14), large controllability energy is needed if $\lambda_{k}$ is small especially when a large component in the direction of $p_{k}$ shall be reached (a component on or outside the unit sphere). This implies that difficult to reach states have a "large" component in the eigenspaces of $P$ belonging to the small eigenvalues.

Remark. We can replace $P$ by the stochastic type II Gramian $P_{2}$ which satisfies (8). This results in the following inequality

$$
\begin{aligned}
x^{T}(t) P_{2}^{-1} x(t) \leq & {\left[\int_{0}^{t} x^{T}(s)\left(A^{T} P_{2}^{-1}+P_{2}^{-1} A+\sum_{i=1}^{m} N_{i}^{T} P_{2}^{-1} N_{i}\right) x(s) d s\right.} \\
& \left.+2 \int_{0}^{t} x^{T}(s) P_{2}^{-1} B u(s) d s+\int_{0}^{t} x^{T}(s) P_{2}^{-1} x(s)\|u(s)\|_{2}^{2} d s\right] .
\end{aligned}
$$

Inserting (8), we then see that

$$
x^{T}(t) P_{2}^{-1} x(t) \leq \int_{0}^{t}\|u(s)\|_{2}^{2} d s+\int_{0}^{t} x^{T}(s) P_{2}^{-1} x(s)\|u(s)\|_{2}^{2} d s .
$$

By Gronwall's inequality, we obtain

$$
x^{T}(t) P_{2}^{-1} x(t) \leq \int_{0}^{t}\|u(s)\|_{2}^{2} d s \mathrm{e}^{\int_{0}^{t}\|u(s)\|_{2}^{2} d s}
$$

which leads to inequality (14) with an additional exponential term but in this case no bound on the control is assumed. Let us suppose that $\|u\|_{L_{T}^{2}} \leq 1$ in case $P_{2}$ is used. Then, a small eigenvalue $\lambda_{2, k}$ of $P_{2}$ implies that $\left\langle x(t, 0, u), p_{2, k}\right\rangle_{2} \quad\left(p_{2, k}\right.$ is the corresponding eigenvector) is small. This means that no large component in the direction of $p_{2, k}$ can be reached with a small control.

Let us now turn our attention to the type II Gramian $Q$. We shorten the notation again and write $x_{x_{0}}(t)$ instead of $x\left(t, x_{0}, u\right)$. The product rule yields

$$
x_{x_{0}}^{T}(t) Q x_{x_{0}}(t)-x_{0}^{T} Q x_{0}=\int_{0}^{t} x_{x_{0}}^{T}(s) Q d x_{x_{0}}(s)+\int_{0}^{t} d x_{x_{0}}^{T}(s) Q x_{x_{0}}(s),
$$

where $t \in[0, T]$. Following the steps from (13) onwards, we obtain

$$
\begin{gathered}
x_{x_{0}}^{T}(t) Q x_{x_{0}}(t)-x_{0}^{T} Q x_{0} \leq\left[\int_{0}^{t} x_{x_{0}}^{T}(s)\left(A^{T} Q+Q A+\sum_{i=1}^{m} N_{i}^{T} Q N_{i}+k^{2} Q\right) x_{x_{0}}(s) d s\right. \\
\left.+2 \int_{0}^{t} x_{x_{0}}^{T}(s) Q B u(s) d s\right] .
\end{gathered}
$$

We insert (10) and evaluate the functions at the final time which gives

$$
\int_{0}^{T}\left\|y\left(s, x_{0}, u\right)\right\|_{2}^{2} d s \leq x_{0}^{T} Q x_{0}+2 \int_{0}^{T} x\left(s, x_{0}, u\right)^{T} Q B u(s) d s .
$$


As in (7) we assume $B=0$. This is a natural choice since in the observability problem an unknown initial condition shall be reconstructed from the observations $y$. Since $B u$ is a term that does not depend on $x_{0}$, it can be assumed to be known and hence be neglected in the considerations. With $B=0$ and (15) we see that the states which produce little observation energy are close to the kernel of $Q$. They are contained in the eigenspaces of $Q$ corresponding to the small eigenvalues.

Remark. If we use $Q_{1}$ which satisfies (8) instead of $Q$, we get an extra term in the energy bound but in this case there is no bound on $u$. So, we have

$$
\begin{aligned}
\int_{0}^{T}\left\|y\left(s, x_{0}, u\right)\right\|_{2}^{2} d s \leq & x_{0}^{T} Q_{1} x_{0}+2 \int_{0}^{T} x\left(s, x_{0}, u\right)^{T} Q_{1} B u(s) d s \\
& +\int_{0}^{T} x\left(s, x_{0}, u\right)^{T} Q_{1} x\left(s, x_{0}, u\right)\|u(s)\|_{2}^{2} d s .
\end{aligned}
$$

Now, if we say that the control $u$ is small, we can conclude that the observation energy is small if the initial state is close to the kernel of $Q_{1}$.

\section{Type II balanced truncation}

Before considering an $\mathcal{H}_{\infty}$ - error bound for BT based on the type II Gramians $P$ and $Q$, we summarise the theory of balancing which is similar to the deterministic linear case [1, 20].

States that are difficult to reach can be characterised by $P$, cf. (14). These states have large components in the span of the eigenvectors corresponding to small eigenvalues of the reachability Gramian $P$. Similarly, states that are difficult to observe are the ones that have large components in the span of eigenvectors corresponding to small eigenvalues of the observability Gramian $Q$, see (15). Now, balancing a system relies on the idea to create a system, where dominant reachable and observable states are the same, i.e., reachability and observability Gramians are simultaneously transformed such that they are equal and diagonal. BT for bilinear systems based on the Gramians $P_{1}$ and $Q_{1}$ (type I ansatz) has already been studied [4] and for related energy functionals compare [15]. For type I BT no error bound could be shown so far. Now, the procedure for the type II approach is explained. This ansatz allows us to show an $\mathcal{H}_{\infty}$-error bound in Section 4 , (2)

We consider a control system consisting of state equation (1) and output equation

$$
\begin{aligned}
\frac{d x(t)}{d t} & =A x(t)+B u(t)+\sum_{i=1}^{m} N_{i} x(t) u_{i}(t), \\
y(t) & =C x(t), \quad t \geq 0,
\end{aligned}
$$

Recall that the state equation in (16) is asymptotically stable, i.e., $\sigma\left(A+\frac{k^{2}}{2}\right) \subset \mathbb{C}_{-}$or ideally (12) is satisfied. Introducing a transformation matrix $T \in \mathbb{R}^{n \times n}$ which is assumed 
to be non-singular, the states are transformed as follows:

$$
\hat{x}(t)=T x(t),
$$

such that system (16) becomes

$$
\begin{aligned}
\frac{d}{d t} \hat{x}(t) & =\hat{A} \hat{x}(t)+\hat{B} u(t)+\sum_{i=1}^{m} \hat{N}_{i} x(t) u_{i}(t), \\
y(t) & =\hat{C} \hat{x}(t), \quad t \geq 0
\end{aligned}
$$

where $\hat{A}=T A T^{-1}, \hat{B}=T B, \hat{C}=C T^{-1}$ and $\hat{N}_{i}=T N_{i} T^{-1}$. The input-output map remains the same, only the state and the systems matrices are transformed.

$P$ and $Q$, the reachability and observability Gramians of system (16), which satisfy (91) and (10) can be transformed into reachability and observability Gramians of the transformed system (17):

$$
\hat{P}=T P T^{T} \text { and } \hat{Q}=T^{-T} Q T^{-1} .
$$

The above relation is obtained by multiplying (9) and (10) with $T^{-T}$ from the left and $T^{-1}$ from the right. The Hankel singular values (HSVs) $\sigma_{1} \geq \ldots \geq \sigma_{n}$, where $\sigma_{i}=\sqrt{\lambda_{i}(P Q)}(i=1, \ldots, n)$, of the original and transformed system are the same. The above transformation is a balancing transformation if the transformed Gramians are equal and diagonal. Such a transformation always exists if $Q>0$ (observation energy is always non zero for every $x_{0} \neq 0$ ). We also need that $P>0$ but this is automatically satisfied. A balanced system is obtained by choosing

$$
T=\Sigma^{-\frac{1}{2}} U^{T} L^{T} \quad \text { and } \quad T^{-1}=K V \Sigma^{-\frac{1}{2}},
$$

where $\Sigma=\operatorname{diag}\left(\sigma_{1}, \ldots, \sigma_{n}\right)>0$ is the diagonal matrix of HSVs. $Y, Z, L$ and $K$ are computed as follows. Let $P=K K^{T}, Q=L L^{T}$ be square root factorisations of $P$ and $Q$, then an SVD of $K^{T} L=V \Sigma U^{T}$ gives the required matrices. With this transformation $\hat{P}=\hat{Q}=\Sigma$. This implies that $\Sigma$ characterises both the reachability and observability in system (17). The smaller the diagonal entry of $\Sigma$, the less important the corresponding state component in the system dynamics of (17).

Below, let $T$ be the balancing transformation as stated above, then we partition the coefficients of the balanced realisation as follows:

$$
T A T^{-1}=\left[\begin{array}{ll}
A_{11} & A_{12} \\
A_{21} & A_{22}
\end{array}\right], \quad T B=\left[\begin{array}{l}
B_{1} \\
B_{2}
\end{array}\right], \quad C T^{-1}=\left[\begin{array}{ll}
C_{1} C_{2}
\end{array}\right], \quad T N_{i} T^{-1}=\left[\begin{array}{ll}
N_{i, 11} & N_{i, 12} \\
N_{i, 21} & N_{i, 22}
\end{array}\right],
$$

where $A_{11} \in \mathbb{R}^{r \times r}$ etc. Furthermore, by setting $\hat{x}=\left[\begin{array}{l}x_{1} \\ x_{2}\end{array}\right]$, where $x_{1}(t) \in \mathbb{R}^{r}$, we obtain the transformed partitioned system

$$
\begin{aligned}
\frac{d}{d t}\left[\begin{array}{l}
x_{1}(t) \\
x_{2}(t)
\end{array}\right] & =\left[\begin{array}{ll}
A_{11} & A_{12} \\
A_{21} & A_{22}
\end{array}\right]\left[\begin{array}{l}
x_{1}(t) \\
x_{2}(t)
\end{array}\right]+\left[\begin{array}{l}
B_{1} \\
B_{2}
\end{array}\right] u(t)+\sum_{i=1}^{m}\left[\begin{array}{ll}
N_{i, 11} & N_{i, 12} \\
N_{i, 21} & N_{i, 22}
\end{array}\right]\left[\begin{array}{l}
x_{1}(t) \\
x_{2}(t)
\end{array}\right] u_{i}(t), \\
y(t) & =\left[\begin{array}{ll}
C_{1} & C_{2}
\end{array}\right]\left[\begin{array}{l}
x_{1}(t) \\
x_{2}(t)
\end{array}\right], t \geq 0 .
\end{aligned}
$$


From this system we aim to obtain a approximating system with reduced dimension $r \ll n$. For BT the second row in (18) is truncated and the remaining $x_{2}$ components in the first row of (18) and in (19) are set to zero. This leads to a ROM having the same structure as (16), that is,

$$
\begin{aligned}
\frac{d x_{r}(t)}{d t} & =A_{11} x_{r}(t)+B_{1} u(t)+\sum_{i=1}^{m} N_{i, 11} x_{r}(t) u_{i}(t), \\
y_{r}(t) & =C_{1} x_{r}(t), \quad t \geq 0,
\end{aligned}
$$

where $A_{11}, N_{i, 11} \in \mathbb{R}^{r \times r}, B_{1} \in \mathbb{R}^{r \times m}$ and $C_{1} \in \mathbb{R}^{p \times r}$. In equations (18) and (19), the difficult to reach and observe states are represented by $x_{2}$, which correspond to the smallest HSVs $\sigma_{r+1}, \ldots, \sigma_{n}$, but of course $r$ has to be chosen such that the neglected HSVs are small $\left(\sigma_{r+1} \ll \sigma_{r}\right)$.

\section{$4 \mathcal{H}_{\infty}$-error bound for type II BT}

In this section, we show that the $\mathcal{H}_{\infty}$-error bound, that is known from the linear case [1], is also true for bilinear systems when using the type II approach. Unfortunately, this result could no yet be established when using the type I Gramians.

We recall the original model that we aim to reduce:

$$
\begin{aligned}
\frac{d x(t)}{d t} & =A x(t)+B u(t)+\sum_{i=1}^{m} N_{i} x(t) u_{i}(t), \quad x(0)=0, \\
y(t) & =C x(t), \quad t \geq 0,
\end{aligned}
$$

where the matrices and vectors above are partitioned as follows

$$
A=\left[\begin{array}{ll}
A_{11} & A_{12} \\
A_{21} & A_{22}
\end{array}\right], x=\left[\begin{array}{l}
x_{1} \\
x_{2}
\end{array}\right], B=\left[\begin{array}{l}
B_{1} \\
B_{2}
\end{array}\right], N_{i}=\left[\begin{array}{ll}
N_{i, 11} & N_{i, 12} \\
N_{i, 21} & N_{i, 22}
\end{array}\right], C=\left[\begin{array}{ll}
C_{1} & C_{2}
\end{array}\right] .
$$

Below, we prove an $\mathcal{H}_{\infty}$-error bound when the balancing relies on the matrix (in)equalities (9) and (10). We replace equation (10) by an inequality because we do not need the equality in the proof. To simplify the notation, we assume that system (21) is balanced already, i.e., we have applied the balancing transformation from Section 3 already. Hence, the Gramians $P$ and $Q$ are equal and coincide with the diagonal matrix $\Sigma=\operatorname{diag}\left(\Sigma_{1}, \Sigma_{2}\right)$, where $\Sigma_{1}=\operatorname{diag}\left(\sigma_{1}, \ldots, \sigma_{r}\right)$ is the matrix of large and $\Sigma_{2}=\operatorname{diag}\left(\sigma_{r+1}, \ldots, \sigma_{n}\right)$ the matrix of neglected small HSVs.

The following ROM is supposed to be compared with the original model (21):

$$
\begin{aligned}
\frac{d x_{r}(t)}{d t} & =A_{11} x_{r}(t)+B_{1} u(t)+\sum_{i=1}^{m} N_{i, 11} x_{r}(t) u_{i}(t), \quad x_{r}(0)=0, \\
y_{r}(t) & =C_{1} x_{r}(t), \quad t \geq 0 .
\end{aligned}
$$

The next theorem deals with the $L_{T}^{2}$-error between the full and the reduced order output. 
Theorem 4.1. Let $x(0)=0, x_{r}(0)=0$ and $\|u(t)\|_{2} \leq k, t \in[0, T], T>0$, where $k$ is the constant that enters in (9) and (10). Then,

$$
\left\|y-y_{r}\right\|_{L_{T}^{2}} \leq 2\left(\tilde{\sigma}_{1}+\tilde{\sigma}_{2}+\ldots+\tilde{\sigma}_{\nu}\right)\|u\|_{L_{T}^{2}},
$$

where $y$ is the output of the original system (21), $y_{r}$ is the output of the type II BT approach $R O M$ and $\tilde{\sigma}_{1}, \tilde{\sigma}_{2}, \ldots, \tilde{\sigma}_{\nu}$ are the distinct diagonal entries of $\Sigma_{2}=\operatorname{diag}\left(\sigma_{r+1}, \ldots, \sigma_{n}\right)=$ $\operatorname{diag}\left(\tilde{\sigma}_{1} I, \tilde{\sigma}_{2} I, \ldots, \tilde{\sigma}_{\nu} I\right)$.

Proof. We sometimes omit the time dependence of the functions in this proof to keep the notation as easy as possible. Inserting for $y$ and $y_{r}$ yields

$$
-\left\|y-y_{r}\right\|_{2}^{2}=-\left\|C_{1}\left(x_{1}-x_{r}\right)+C_{2} x_{2}\right\|_{2}^{2}=-\left[\begin{array}{c}
x_{1}-x_{r} \\
x_{2}
\end{array}\right]^{T} C^{T} C\left[\begin{array}{c}
x_{1}-x_{r} \\
x_{2}
\end{array}\right] .
$$

The partitioned matrix (in)equality (10)

$$
\begin{aligned}
& {\left[\begin{array}{ll}
A_{11} & A_{12} \\
A_{21} & A_{22}
\end{array}\right]^{T}\left[\begin{array}{ll}
\Sigma_{1} & \\
& \Sigma_{2}
\end{array}\right]+\left[\begin{array}{ll}
\Sigma_{1} & \\
& \Sigma_{2}
\end{array}\right]\left[\begin{array}{ll}
A_{11} & A_{12} \\
A_{21} & A_{22}
\end{array}\right]+\sum_{i=1}^{m}\left[\begin{array}{ll}
N_{i, 11} & N_{i, 12} \\
N_{i, 21} & N_{i, 22}
\end{array}\right]^{T}\left[\begin{array}{ll}
\Sigma_{1} & \\
& \Sigma_{2}
\end{array}\right]\left[\begin{array}{ll}
N_{i, 11} & N_{i, 12} \\
N_{i, 21} & N_{i, 22}
\end{array}\right]+k^{2}\left[\begin{array}{ll}
\Sigma_{1} & \\
& \Sigma_{2}
\end{array}\right]} \\
& \leq-C^{T} C
\end{aligned}
$$

leads to

$$
\begin{aligned}
& -\left\|y-y_{r}\right\|_{2}^{2} \geq \\
& 2\left(x_{1}-x_{r}\right)^{T} \Sigma_{1}\left[\begin{array}{ll}
A_{11} & A_{12}
\end{array}\right]\left[\begin{array}{c}
x_{1}-x_{r} \\
x_{2}
\end{array}\right]+\sum_{i=1}^{m}\left(\left[N_{i, 11} N_{i, 12}\right]\left[\begin{array}{c}
x_{1}-x_{r} \\
x_{2}
\end{array}\right]\right)^{T} \Sigma_{1}\left[\begin{array}{ll}
N_{i, 11} & N_{i, 12}
\end{array}\right]\left[\begin{array}{c}
x_{1}-x_{r} \\
x_{2}
\end{array}\right] \\
& +\left(x_{1}-x_{r}\right)^{T} \Sigma_{1} k^{2}\left(x_{1}-x_{r}\right)+x_{2}^{T} \Sigma_{2} k^{2} x_{2} \\
& +2 x_{2}^{T} \Sigma_{2}\left[\begin{array}{ll}
A_{21} & A_{22}
\end{array}\right]\left[\begin{array}{c}
x_{1}-x_{r} \\
x_{2}
\end{array}\right]+\sum_{i=1}^{m}\left(\left[\begin{array}{cc}
N_{i, 21} & N_{i, 22}
\end{array}\right]\left[\begin{array}{c}
x_{1}-x_{r} \\
x_{2}
\end{array}\right]\right)^{T} \Sigma_{2}\left[\begin{array}{ll}
N_{i, 21} & N_{i, 22}
\end{array}\right]\left[\begin{array}{c}
x_{1}-x_{r} \\
x_{2}
\end{array}\right] .
\end{aligned}
$$

Using the above summands, we define

$$
\begin{aligned}
& \mathcal{T}_{1}:=2\left(x_{1}-x_{r}\right)^{T} \Sigma_{1}\left[\begin{array}{ll}
A_{11} & A_{12}
\end{array}\right]\left[\begin{array}{c}
x_{1}-x_{r} \\
x_{2}
\end{array}\right], \\
& \mathcal{T}_{2}:=\sum_{i=1}^{m}\left(\left[\begin{array}{ll}
N_{i, 11} & N_{i, 12}
\end{array}\right]\left[\begin{array}{c}
x_{1}-x_{r} \\
x_{2}
\end{array}\right]\right)^{T} \Sigma_{1}\left[\begin{array}{ll}
N_{i, 11} & N_{i, 12}
\end{array}\right]\left[\begin{array}{c}
x_{1}-x_{r} \\
x_{2}
\end{array}\right]+\left(x_{1}-x_{r}\right)^{T} \Sigma_{1} k^{2}\left(x_{1}-x_{r}\right), \\
& \mathcal{T}_{3}:=2 x_{2}^{T} \Sigma_{2}\left[\begin{array}{ll}
A_{21} & A_{22}
\end{array}\right]\left[\begin{array}{c}
x_{1}-x_{r} \\
x_{2}
\end{array}\right], \\
& \mathcal{T}_{4}:=\sum_{i=1}^{m}\left(\left[\begin{array}{ll}
N_{i, 21} & N_{i, 22}
\end{array}\right]\left[\begin{array}{c}
x_{1}-x_{r} \\
x_{2}
\end{array}\right]\right)^{T} \Sigma_{2}\left[\begin{array}{ll}
N_{i, 21} & N_{i, 22}
\end{array}\right]\left[\begin{array}{c}
x_{1}-x_{r} \\
x_{2}
\end{array}\right]+x_{2}^{T} \Sigma_{2} k^{2} x_{2} .
\end{aligned}
$$

The differential of $x_{1}-x_{r}$ is given by

$$
\frac{d\left(x_{1}-x_{r}\right)}{d t}=\left[\begin{array}{ll}
A_{11} & A_{12}
\end{array}\right]\left[\begin{array}{c}
x_{1}-x_{r} \\
x_{2}
\end{array}\right]+\sum_{i=1}^{m}\left[\begin{array}{ll}
N_{i, 11} & N_{i, 12}
\end{array}\right]\left[\begin{array}{c}
x_{1}-x_{r} \\
x_{2}
\end{array}\right] u_{i}
$$


which we insert into the following definition:

$$
D_{1}:=\frac{d\left(\left(x_{1}(t)-x_{r}(t)\right)^{T} \Sigma_{1}\left(x_{1}(t)-x_{r}(t)\right)\right)}{d t}=2\left(x_{1}(t)-x_{r}(t)\right)^{T} \Sigma_{1} \frac{d\left(x_{1}(t)-x_{r}(t)\right)}{d t} .
$$

Hence, we have

$$
D_{1}=2\left(x_{1}-x_{r}\right)^{T} \Sigma_{1}\left(\left[\begin{array}{ll}
A_{11} & A_{12}
\end{array}\right]\left[\begin{array}{c}
x_{1}-x_{r} \\
x_{2}
\end{array}\right]+\sum_{i=1}^{m}\left[\begin{array}{ll}
N_{i, 11} & N_{i, 12}
\end{array}\right]\left[\begin{array}{c}
x_{1}-x_{r} \\
x_{2}
\end{array}\right] u_{i}\right) .
$$

We use an elementary estimate for the following inner product

$$
\begin{aligned}
& \sum_{i=1}^{m} 2\left(x_{1}-x_{r}\right)^{T} \Sigma_{1}\left[\begin{array}{ll}
N_{i, 11} & N_{i, 12}
\end{array}\right]\left[\begin{array}{c}
x_{1}-x_{r} \\
x_{2}
\end{array}\right] u_{i} \\
& =\sum_{i=1}^{m} 2\left\langle\Sigma_{1}^{\frac{1}{2}}\left(x_{1}-x_{r}\right) u_{i}, \Sigma_{1}^{\frac{1}{2}}\left[N_{i, 11} N_{i, 12}\right]\left[\begin{array}{c}
x_{1}-x_{r} \\
x_{2}
\end{array}\right]\right\rangle_{2}
\end{aligned}
$$

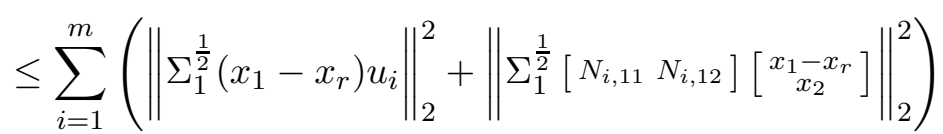

$$
\begin{aligned}
& =\left(x_{1}-x_{r}\right)^{T} \Sigma_{1}\left(x_{1}-x_{r}\right)\|u\|_{2}^{2}+\sum_{i=1}^{m}\left(\left[N_{i, 11} N_{i, 12}\right]\left[\begin{array}{c}
x_{1}-x_{r} \\
x_{2}
\end{array}\right]\right)^{T} \Sigma_{1}\left[N_{i, 11} N_{i, 12}\right]\left[\begin{array}{c}
x_{1}-x_{r} \\
x_{2}
\end{array}\right] .
\end{aligned}
$$

Using the fact that $u$ is bounded, i.e., $\|u\|_{2} \leq k$ then yields

$$
\frac{d}{d t}\left(x_{1}(t)-x_{r}(t)\right)^{T} \Sigma_{1}\left(x_{1}(t)-x_{r}(t)\right) \leq \mathcal{T}_{1}+\mathcal{T}_{2} .
$$

From (21) the variable $x_{2}$ satifies

$$
\frac{d x_{2}(t)}{d t}=\left[\begin{array}{ll}
A_{21} & A_{22}
\end{array}\right]\left[\begin{array}{l}
x_{1}(t) \\
x_{2}(t)
\end{array}\right]+B_{2} u(t)+\sum_{i=1}^{m}\left[\begin{array}{ll}
N_{i, 21} & N_{i, 22}
\end{array}\right]\left[\begin{array}{l}
x_{1}(t) \\
x_{2}(t)
\end{array}\right] u_{i}(t) .
$$

Plugging this into $\frac{d x_{2}(t)^{T} \Sigma_{2} x_{2}(t)}{d t}=2 x_{2}(t)^{T} \Sigma_{2} \frac{x_{2}(t)}{d t}$ provides the following relation

$$
\frac{d x_{2}}{d t}=2 x_{2}^{T} \Sigma_{2}\left(\left[\begin{array}{ll}
A_{21} & A_{22}
\end{array}\right]\left[\begin{array}{l}
x_{1} \\
x_{2}
\end{array}\right]+B_{2} u+\sum_{i=1}^{m}\left[\begin{array}{ll}
N_{i, 21} & N_{i, 22}
\end{array}\right]\left[\begin{array}{l}
x_{1} \\
x_{2}
\end{array}\right] u_{i}\right) .
$$

We find an upper bound for the last term the same way we have done before

$$
\begin{aligned}
& \sum_{i=1}^{m} 2 x_{2}^{T} \Sigma_{2}\left[\begin{array}{ll}
N_{i, 21} & N_{i, 22}
\end{array}\right]\left[\begin{array}{c}
x_{1} \\
x_{2}
\end{array}\right] u_{i}=\sum_{i=1}^{m} 2\left\langle\Sigma_{2}^{\frac{1}{2}} x_{2} u_{i}, \Sigma_{2}^{\frac{1}{2}}\left[\begin{array}{ll}
N_{i, 21} & N_{i, 22}
\end{array}\right]\left[\begin{array}{l}
x_{1} \\
x_{2}
\end{array}\right]\right\rangle_{2} \\
& \leq x_{2}^{T} \Sigma_{2} x_{2} k^{2}+\sum_{i=1}^{m}\left(\left[\begin{array}{ll}
N_{i, 21} & N_{i, 22}
\end{array}\right]\left[\begin{array}{l}
x_{1} \\
x_{2}
\end{array}\right]\right)^{T} \Sigma_{2}\left[\begin{array}{ll}
N_{i, 21} & N_{i, 22}
\end{array}\right]\left[\begin{array}{l}
x_{1} \\
x_{2}
\end{array}\right]
\end{aligned}
$$


applying again that the control is bounded by $k$. This yields

$$
\begin{aligned}
& \frac{d}{d t}\left(x_{2}(t)^{T} \Sigma_{2} x_{2}(t)\right) \leq\left[\mathcal{T}_{3}+2 x_{2}^{T} \Sigma_{2}\left(A_{21} x_{r}+B_{2} u\right)\right] \\
& +\left[\mathcal{T}_{4}+2 \sum_{i=1}^{m}\left(N_{i, 21} x_{r}\right)^{T} \Sigma_{2}\left[\begin{array}{ll}
N_{i, 21} & N_{i, 22}
\end{array}\right]\left[\begin{array}{l}
x_{1} \\
x_{2}
\end{array}\right]\right. \\
& \left.-\sum_{i=1}^{m}\left(N_{i, 21} x_{r}\right)^{T} \Sigma_{2} N_{i, 21} x_{r}\right] .
\end{aligned}
$$

Summarising the above computations, we obtain

$$
\begin{aligned}
-\left\|y-y_{r}\right\|_{2}^{2} & \geq \mathcal{T}_{1}+\mathcal{T}_{2}+\mathcal{T}_{3}+\mathcal{T}_{4} \geq \frac{d}{d t}\left(\left(x_{1}-x_{r}\right)^{T} \Sigma_{1}\left(x_{1}-x_{r}\right)\right)+\frac{d}{d t}\left(x_{2}^{T} \Sigma_{2} x_{2}\right) \\
& -2 x_{2}^{T} \Sigma_{2}\left(A_{21} x_{r}+B_{2} u\right)-2 \sum_{i=1}^{m}\left(N_{i, 21} x_{r}\right)^{T} \Sigma_{2}\left[\begin{array}{ll}
N_{i, 21} & N_{i, 22}
\end{array}\right]\left[\begin{array}{l}
x_{1} \\
x_{2}
\end{array}\right] .
\end{aligned}
$$

For the moment we assume that $\Sigma_{2}=\sigma I$. Since we have zero initial conditions, it holds that

$$
\begin{aligned}
\int_{0}^{T}\left\|y(t)-y_{r}(t)\right\|_{2}^{2} d t \leq 2 \sigma^{2}( & \int_{0}^{T} x_{2}^{T} \Sigma_{2}^{-1}\left(A_{21} x_{r}+B_{2} u\right) d t \\
& \left.+\int_{0}^{T} \sum_{i=1}^{m}\left(N_{i, 21} x_{r}\right)^{T} \Sigma_{2}^{-1}\left[\begin{array}{ll}
N_{i, 21} & N_{i, 22}
\end{array}\right]\left[\begin{array}{l}
x_{1} \\
x_{2}
\end{array}\right] d t\right) .
\end{aligned}
$$

Inequality (91) and the Schur complement condition on definiteness imply

$$
\left[\begin{array}{cc}
A^{T} \Sigma^{-1}+\Sigma^{-1} A+\sum_{i=1}^{m} N_{i}^{T} \Sigma^{-1} N_{i}+\Sigma^{-1} k^{2} & \Sigma^{-1} B \\
B^{T} \Sigma^{-1} & -I
\end{array}\right] \leq 0 .
$$

If we multiply the matrix inequality (27) with $\left[\begin{array}{c}x_{1}+x_{r} \\ x_{2} \\ 2 u\end{array}\right]^{T}$ from the left and with $\left[\begin{array}{c}x_{1}+x_{r} \\ x_{2} \\ 2 u\end{array}\right]$ from the right, we get

$$
\begin{aligned}
4\|u\|_{2}^{2} \geq & 2\left(x_{1}+x_{r}\right)^{T} \Sigma_{1}^{-1}\left(\left[\begin{array}{ll}
A_{11} & A_{12}
\end{array}\right]\left[\begin{array}{c}
x_{1}+x_{r} \\
x_{2}
\end{array}\right]+2 B_{1} u\right)+\left(x_{1}-x_{r}\right)^{T} \Sigma_{1}^{-1} k^{2}\left(x_{1}-x_{r}\right) \\
& +\sum_{i=1}^{m}\left(\left[\begin{array}{ll}
N_{i, 11} & N_{i, 12}
\end{array}\right]\left[\begin{array}{c}
x_{1}+x_{r} \\
x_{2}
\end{array}\right]\right)^{T} \Sigma_{1}^{-1}\left[\begin{array}{ll}
N_{i, 11} & N_{i, 12}
\end{array}\right]\left[\begin{array}{c}
x_{1}+x_{r} \\
x_{2}
\end{array}\right] \\
& +2 x_{2}^{T} \Sigma_{2}^{-1}\left(\left[\begin{array}{ll}
A_{21} & A_{22}
\end{array}\right]\left[\begin{array}{c}
x_{1}+x_{r} \\
x_{2}
\end{array}\right]+2 B_{2} u\right)+x_{2}^{T} \Sigma_{2}^{-1} k^{2} x_{2} \\
& +\sum_{i=1}^{m}\left(\left[\begin{array}{ll}
N_{i, 21} & N_{i, 22}
\end{array}\right]\left[\begin{array}{cc}
x_{1}+x_{r} \\
x_{2}
\end{array}\right]\right)^{T} \Sigma_{2}^{-1}\left[\begin{array}{ll}
N_{i, 21} & N_{i, 22}
\end{array}\right]\left[\begin{array}{c}
x_{1}+x_{r} \\
x_{2}
\end{array}\right] .
\end{aligned}
$$

The above terms are used to define

$$
\mathcal{T}_{5}:=2\left(x_{1}+x_{r}\right)^{T} \Sigma_{1}^{-1}\left(\left[\begin{array}{ll}
A_{11} & A_{12}
\end{array}\right]\left[\begin{array}{c}
x_{1}+x_{r} \\
x_{2}
\end{array}\right]+2 B_{1} u\right),
$$




$$
\begin{aligned}
& \mathcal{T}_{6}:=\left(x_{1}+x_{r}\right)^{T} \Sigma_{1}^{-1} k^{2}\left(x_{1}+x_{r}\right)+\sum_{i=1}^{m}\left(\left[N_{i, 11} N_{i, 12}\right]\left[\begin{array}{c}
x_{1}+x_{r} \\
x_{2}
\end{array}\right]\right)^{T} \Sigma_{1}^{-1}\left[\begin{array}{ll}
N_{i, 11} & N_{i, 12}
\end{array}\right]\left[\begin{array}{c}
x_{1}+x_{r} \\
x_{2}
\end{array}\right], \\
& \mathcal{T}_{7}:=2 x_{2}^{T} \Sigma_{2}^{-1}\left(\left[\begin{array}{ll}
A_{21} & A_{22}
\end{array}\right]\left[\begin{array}{c}
x_{1}+x_{r} \\
x_{2}
\end{array}\right]+2 B_{2} u\right), \\
& \mathcal{T}_{8}:=x_{2}^{T} \Sigma_{2}^{-1} k^{2} x_{2}+\sum_{i=1}^{m}\left(\left[\begin{array}{cc}
N_{i, 21} & N_{i, 22}
\end{array}\right]\left[\begin{array}{c}
x_{1}+x_{r} \\
x_{2}
\end{array}\right]\right)^{T} \Sigma_{2}^{-1}\left[N_{i, 21} N_{i, 22}\right]\left[\begin{array}{c}
x_{1}+x_{r} \\
x_{2}
\end{array}\right] .
\end{aligned}
$$

Exploiting the following equation

$$
\begin{aligned}
\frac{d\left(x_{1}(t)+x_{r}(t)\right)}{d t}= & {\left[\begin{array}{ll}
A_{11} & A_{12}
\end{array}\right]\left[\begin{array}{c}
x_{1}(t)+x_{r}(t) \\
x_{2}(t)
\end{array}\right]+2 B_{1} u(t) } \\
& +\sum_{i=1}^{m}\left[\begin{array}{ll}
N_{i, 11} & N_{i, 12}
\end{array}\right]\left[\begin{array}{c}
x_{1}(t)+x_{r}(t) \\
x_{2}(t)
\end{array}\right] u_{i}(t),
\end{aligned}
$$

we derive

$$
\begin{aligned}
& \frac{d}{d t}\left(\left(x_{1}+x_{r}\right)^{T} \Sigma_{1}^{-1}\left(x_{1}+x_{r}\right)\right)=2\left(x_{1}+x_{r}\right)^{T} \Sigma_{1}^{-1} \frac{d}{d t}\left(x_{1}+x_{r}\right) \\
& =2\left(x_{1}+x_{r}\right)^{T} \Sigma_{1}^{-1}\left(\left[\begin{array}{ll}
A_{11} & A_{12}
\end{array}\right]\left[\begin{array}{c}
x_{1}+x_{r} \\
x_{2}
\end{array}\right]+2 B_{1} u+\sum_{i=1}^{m}\left[\begin{array}{ll}
N_{i, 11} & N_{i, 12}
\end{array}\right]\left[\begin{array}{c}
{ }_{1}+x_{r} \\
x_{2}
\end{array}\right] u_{i}\right) .
\end{aligned}
$$

Analogously to the above computations the last term can be bounded as follows

$$
\begin{aligned}
& \sum_{i=1}^{m} 2\left(x_{1}+x_{r}\right)^{T} \Sigma_{1}^{-1}\left[N_{i, 11} N_{i, 12}\right]\left[\begin{array}{c}
x_{1}+x_{r} \\
x_{2}
\end{array}\right] u_{i} \\
& =\sum_{i=1}^{m} 2\left\langle\Sigma_{1}^{-\frac{1}{2}}\left(x_{1}+x_{r}\right) u_{i}, \Sigma_{1}^{-\frac{1}{2}}\left[\begin{array}{cc}
N_{i, 11} & N_{i, 12}
\end{array}\right]\left[\begin{array}{c}
x_{1}+x_{r} \\
x_{2}
\end{array}\right]\right\rangle_{2} \\
& \leq\left(x_{1}+x_{r}\right)^{T} \Sigma_{1}^{-1}\left(x_{1}+x_{r}\right) k^{2}+\sum_{i=1}^{m}\left(\left[\begin{array}{ll}
N_{i, 11} & N_{i, 12}
\end{array}\right]\left[\begin{array}{c}
x_{1}+x_{r} \\
x_{2}
\end{array}\right]\right)^{T} \Sigma_{1}^{-1}\left[\begin{array}{ll}
N_{i, 11} & N_{i, 12}
\end{array}\right]\left[\begin{array}{c}
x_{1}+x_{r} \\
x_{2}
\end{array}\right] .
\end{aligned}
$$

This estimate yields

$$
\frac{d}{d t}\left(\left(x_{1}(t)+x_{r}(t)\right)^{T} \Sigma_{1}^{-1}\left(x_{1}(t)+x_{r}(t)\right)\right) \leq \mathcal{T}_{5}+\mathcal{T}_{6} .
$$

With equation (24) and the previous steps, we know that

$$
\begin{aligned}
\frac{d\left(x_{2}^{T} \Sigma_{2}^{-1} x_{2}\right)}{d t} \leq & 2 x_{2}^{T} \Sigma_{2}^{-1}\left(\left[\begin{array}{ll}
A_{21} & A_{22}
\end{array}\right]\left[\begin{array}{c}
x_{1} \\
x_{2}
\end{array}\right]+B_{2} u\right)+x_{2}^{T} \Sigma_{2}^{-1} x_{2} k^{2} \\
& +\sum_{i=1}^{m}\left(\left[\begin{array}{ll}
N_{i, 21} & N_{i, 22}
\end{array}\right]\left[\begin{array}{l}
x_{1} \\
x_{2}
\end{array}\right]\right)^{T} \Sigma_{2}^{-1}\left[\begin{array}{ll}
N_{i, 21} & N_{i, 22}
\end{array}\right]\left[\begin{array}{c}
x_{1} \\
x_{2}
\end{array}\right],
\end{aligned}
$$


such that

$$
\begin{aligned}
& \frac{d}{d t}\left(x_{2}(t)^{T} \Sigma_{2}^{-1} x_{2}(t)\right) \leq\left[\mathcal{T}_{7}-2 x_{2}^{T} \Sigma_{2}^{-1}\left(A_{21} x_{r}+B_{2} u\right)\right] \\
& +\left[\mathcal{T}_{8}-2 \sum_{i=1}^{m}\left(N_{i, 21} x_{r}\right)^{T} \Sigma_{2}^{-1}\left[\begin{array}{ll}
N_{i, 21} & N_{i, 22}
\end{array}\right]\left[\begin{array}{l}
x_{1} \\
x_{2}
\end{array}\right]\right. \\
& \left.-\sum_{i=1}^{m}\left(N_{i, 21} x_{r}\right)^{T} \Sigma_{2}^{-1} N_{i, 21} x_{r}\right] .
\end{aligned}
$$

In summary, we obtain

$$
\begin{aligned}
4\|u(t)\|_{2}^{2} \geq & \mathcal{T}_{5}+\mathcal{T}_{6}+\mathcal{T}_{7}+\mathcal{T}_{8} \\
\geq & \frac{d}{d t}\left(\left(x_{1}(t)+x_{r}(t)\right)^{T} \Sigma_{1}^{-1}\left(x_{1}(t)+x_{r}(t)\right)\right)+\frac{d}{d t}\left(x_{2}(t)^{T} \Sigma_{2}^{-1} x_{2}(t)\right) \\
& +2 x_{2}^{T} \Sigma_{2}^{-1}\left(A_{21} x_{r}+B_{2} u\right)+2 \sum_{i=1}^{m}\left(N_{i, 21} x_{r}\right)^{T} \Sigma_{2}^{-1}\left[N_{i, 21} N_{i, 22}\right]\left[\begin{array}{c}
x_{1} \\
x_{2}
\end{array}\right]
\end{aligned}
$$

Integration of both sides yields

$$
\begin{aligned}
4 \int_{0}^{T}\|u(t)\|_{2}^{2} d t \geq 2[ & \int_{0}^{T} x_{2}^{T} \Sigma_{2}^{-1}\left(A_{21} x_{r}+B_{2} u\right) d t \\
& \left.+\int_{0}^{T} \sum_{i=1}^{m}\left(N_{i, 21} x_{r}\right)^{T} \Sigma_{2}^{-1}\left[N_{i, 21} N_{i, 22}\right]\left[\begin{array}{l}
x_{1} \\
x_{2}
\end{array}\right] d t\right] .
\end{aligned}
$$

Combining this inequality with (26) leads to

$$
\left(\int_{0}^{T}\left\|y(t)-y_{r}(t)\right\|_{2}^{2} d t\right)^{\frac{1}{2}} \leq 2 \sigma\left(\int_{0}^{T}\|u(t)\|_{2}^{2} d t\right)^{\frac{1}{2}} .
$$

The proof for general $\Sigma_{2}$ relies on the common idea of removing the Hankel singular values step by step. The error between the outputs $y$ and $y_{r}$ can be bounded as follows:

$$
\left\|y-y_{r}\right\|_{L_{T}^{2}} \leq\left\|y-y_{r_{\nu}}\right\|_{L_{T}^{2}}+\left\|y_{r_{\nu}}-y_{r_{\nu-1}}\right\|_{L_{T}^{2}}+\ldots+\left\|y_{r_{2}}-y_{r}\right\|_{L_{T}^{2}}
$$

where the dimensions $r_{i}$ of the corresponding states are defined by $r_{i+1}=r_{i}+m\left(\tilde{\sigma}_{i}\right)$ for $i=1,2 \ldots, \nu-1$. Here, $m\left(\tilde{\sigma}_{i}\right)$ denotes the multiplicity of $\tilde{\sigma}_{i}$ and $r_{1}=r$. In the reduction step from $y$ to $y_{r_{\nu}}$ only the smallest Hankel singular value $\tilde{\sigma}_{\nu}$ is removed from the system. Hence, by inequality (31), we have

$$
\left\|y-y_{r_{\nu}}\right\|_{L_{T}^{2}} \leq 2 \tilde{\sigma}_{\nu}\|u\|_{L_{T}^{2}} .
$$

Inequality (31) can be established as well when comparing the reduced order outputs $y_{r_{\nu}}$ and $y_{r_{\nu-1}}$. Again, only one Hankel singular value, namely $\tilde{\sigma}_{r_{\nu-1}}$, is removed. At the 
same time, we have the same kind of inequalities in the ROM as before, that are,

$$
\begin{gathered}
A_{11}^{T} \Sigma_{1}^{-1}+\Sigma_{1}^{-1} A_{11}+\sum_{i=1}^{m} N_{i, 11}^{T} \Sigma_{1}^{-1} N_{i, 11}+\Sigma_{1}^{-1} k^{2} \leq-\Sigma_{1}^{-1} B_{1} B_{1}^{T} \Sigma_{1}^{-1}, \\
A_{11}^{T} \Sigma_{1}+\Sigma_{1} A_{11}+\sum_{i=1}^{m} N_{i, 11}^{T} \Sigma_{1} N_{i, 11}+\Sigma_{1} k^{2} \leq-C_{1}^{T} C_{1} .
\end{gathered}
$$

So, by repeatedly applying the above arguments, we obtain

$$
\left\|y_{r_{j}}-y_{r_{j-1}}\right\|_{L_{T}^{2}} \leq 2 \tilde{\sigma}_{r_{j-1}}\|u\|_{L_{T}^{2}}
$$

for $j=2, \ldots, \nu$. This provides the claimed result.

Since the bound in Theorem 4.1 involves only the sum of distinct diagonal entries of $\Sigma_{2}$, the result is also true when using the sum of all diagonal entries instead.

Corollary 4.2. Let $x(0)=0, x_{r}(0)=0$ and $\|u(t)\|_{2} \leq k, t \in[0, T], T>0$, where $k$ is the constant that enters in (9) and (10). Then,

$$
\left\|y-y_{r}\right\|_{L_{T}^{2}} \leq 2\left(\sigma_{r+1}+\sigma_{r+2}+\ldots+\sigma_{n}\right)\|u\|_{L_{T}^{2}},
$$

where $y$ is the output of the original system (21), $y_{r}$ is the output of the type II BT ROM and $\sigma_{r+1}, \ldots, \sigma_{n}$ are the diagonal entries of $\Sigma_{2}$.

The $\mathcal{H}_{\infty}$-error of using type II BT depends on the $n-r$ smallest HSVs of the original system. If now only states are neglected that correspond to small values $\sigma_{r+1}, \ldots, \sigma_{n}$ (hardly reachable and observable states), BT leads to a good approximation of the full order system by Corollary 4.2 .

Remark. Theorem 4.1 and Corollary 4.2 can also be achieved when balancing based on $Q_{1}$ and $P_{2}$ satisfying (5) and (8), respectively. In this case, the same techniques can be used. The key points are inequalities (25) and (28). Supposing that $\Sigma_{S}=\operatorname{diag}\left(\Sigma_{S, 1}, \Sigma_{S, 2}\right)$ is the matrix of HSVs from $Q_{1}$ and $P_{2}$, (25) then is

$$
\begin{aligned}
-\left\|y-y_{r}\right\|_{2}^{2} \geq & \frac{d}{d t}\left(x_{1}-x_{r}\right)^{T} \Sigma_{S, 1}\left(x_{1}-x_{r}\right)+\frac{d}{d t} x_{2}^{T} \Sigma_{S, 2} x_{2} \\
& -\left(\left(x_{1}-x_{r}\right)^{T} \Sigma_{S, 1}\left(x_{1}-x_{r}\right)\|u\|_{2}^{2}+x_{2}^{T} \Sigma_{S, 2} x_{2}\|u\|_{2}^{2}\right) \\
& -2 x_{2}^{T} \Sigma_{S, 2}\left(A_{21} x_{r}+B_{2} u\right)-2 \sum_{i=1}^{m}\left(N_{i, 21} x_{r}\right)^{T} \Sigma_{S, 2}\left[\begin{array}{ll}
N_{i, 21} & N_{i, 22}
\end{array}\right]\left[\begin{array}{l}
x_{1} \\
x_{2}
\end{array}\right] .
\end{aligned}
$$

In order to find (26) from this, we have to guarantee that

$$
\left[\begin{array}{c}
x_{1}(T)-x_{r}(T) \\
x_{2}(T)
\end{array}\right]^{T} \Sigma_{S}\left[\begin{array}{c}
x_{1}(T)-x_{r}(T) \\
x_{2}(T)
\end{array}\right] \geq \int_{0}^{T}\left[\begin{array}{c}
x_{1}-x_{r} \\
x_{2}
\end{array}\right]^{T} \Sigma_{S}\left[\begin{array}{c}
x_{1}-x_{r} \\
x_{2}
\end{array}\right]^{T}\|u\|_{2}^{2} d s .
$$


With the alternative Gramians (28) reads

$$
\begin{aligned}
4\|u(t)\|_{2}^{2} \geq & \frac{d}{d t}\left(x_{1}+x_{r}\right)^{T} \Sigma_{S, 1}^{-1}\left(x_{1}+x_{r}\right)+\frac{d}{d t} x_{2}^{T} \Sigma_{S, 2}^{-1} x_{2} \\
& -\left(\left(x_{1}+x_{r}\right)^{T} \Sigma_{S, 1}^{-1}\left(x_{1}+x_{r}\right)\|u\|_{2}^{2}+x_{2}^{T} \Sigma_{S, 2}^{-1} x_{2}\|u\|_{2}^{2}\right) \\
& +2 x_{2}^{T} \Sigma_{S, 2}^{-1}\left(A_{21} x_{r}+B_{2} u\right)+2 \sum_{i=1}^{m}\left(N_{i, 21} x_{r}\right)^{T} \Sigma_{S, 2}^{-1}\left[N_{i, 21} N_{i, 22}\right]\left[\begin{array}{l}
x_{1} \\
x_{2}
\end{array}\right] .
\end{aligned}
$$

To obtain (29), it needs to be ensured that

$$
\left[\begin{array}{c}
x_{1}(T)+x_{r}(T) \\
x_{2}(T)
\end{array}\right]^{T} \Sigma_{S}^{-1}\left[\begin{array}{c}
x_{1}(T)+x_{r}(T) \\
x_{2}(T)
\end{array}\right] \geq \int_{0}^{T}\left[\begin{array}{c}
x_{1}+x_{r} \\
x_{2}
\end{array}\right]^{T} \Sigma_{S}^{-1}\left[\begin{array}{c}
x_{1}+x_{r} \\
x_{2}
\end{array}\right]^{T}\|u\|_{2}^{2} d s .
$$

Both (32) and (33) are true if the control $u$ is small enough. Consequently, Theorem 4.1 and Corollary 4.2 hold when using $Q_{1}$ and $P_{2}$ instead if $\|u(t)\|_{2} \leq k_{2}, t \in[0, T]$, for a sufficiently small constant $k_{2}>0$.

\section{Conclusions}

We have summerised previous work on balanced truncation for bilinear control systems. We have discussed Gramians that have been studied before and how they can be used to bound controllability and observability energy functionals, however, these bounds only hold in a small neighbourhood of zero. We proposed new Gramians (type II) which contain additional information about the control. With these type II Gramians global energy bounds can be found if the controls are assumed to be bounded in a certain way. These bounds justify the use of the alternative Gramians in the context of balancing. Based on this motivation, we explained the balancing procedure for bilinear systems which is similar to the one in the linear case. Another advantage of using the type II Gramians is the availability of an $\mathcal{H}_{\infty}$-error bound for balanced truncation of bilinear systems which we proved in this paper. The error bound requires the assumption of having a bounded input to the system. Hence, we have overcome the problem of previous works, where no error bound has been established.

\section{Acknowledgements}

The author thanks the organisers of the LMS-EPSRC Durham Symposium on Model Order Reduction during which most of the work on this paper was carried out.

\section{References}

[1] A. C. Antoulas. Approximation of large-scale dynamical systems. Advances in Design and Control 6. Philadelphia, PA: SIAM, 2005. 
[2] P. Benner and T. Breiten. On $\mathcal{H}_{2}$-model reduction of linear parameter-varying systems. Proc. Appl. Math. Mech., 11:805-806, 2011.

[3] P. Benner and T. Breiten. Interpolation-based $\mathcal{H}_{2}$-model reduction of bilinear control systems. SIAM J. Matrix Anal. Appl., 33(3):859-885, 2012.

[4] P. Benner and T. Damm. Lyapunov equations, energy functionals, and model order reduction of bilinear and stochastic systems. SIAM J. Control Optim., 49(2):686711, 2011.

[5] P. Benner, T. Damm, and Y. R. R. Cruz. Dual pairs of generalized Lyapunov inequalities and balanced truncation of stochastic linear systems. IEEE Trans. Autom. Contr., 62(2):782-791, 2017.

[6] P. Benner, P. Goyal, and M. Redmann. Truncated Gramians for Bilinear Systems and their Advantages in Model Order Reduction. Model Reduction of Parametrized Systems, MS\&A - Modeling, Simulation and Applications, Springer International Publishing, Accepted December 2016.

[7] P. Benner, V. Mehrmann, and D. C. Sorensen (eds.). Dimension reduction of largescale systems. Proceedings of a workshop, Oberwolfach, Germany, October 19-25, 2003. Lecture Notes in Computational Science and Engineering 45. Berlin: Springer, 2005.

[8] P. Benner and M. Redmann. Model Reduction for Stochastic Systems. Stoch PDE: Anal Comp, 3(3):291-338, 2015.

[9] T. Breiten and T. Damm. Krylov subspace methods for model order reduction of bilinear control systems. Systems Control Lett., 59(10):443-450, 2010.

[10] C. Bruni, G. DiPillo, and G. Koch. On the mathematical models of bilinear systems. Automatica, 2(1):11-26, 1971.

[11] T. Damm. Rational Matrix Equations in Stochastic Control. Lecture Notes in Control and Information Sciences 297. Berlin: Springer, 2004.

[12] T. Damm and P. Benner. Balanced truncation for stochastic linear systems with guaranteed error bound. Proceedings of MTNS-2014, Groningen, The Netherlands, pages 1492-1497, 2014.

[13] G. Flagg and S. Gugercin. Multipoint Volterra series interpolation and $\mathcal{H}_{2}$ optimal model reduction of bilinear systems. SIAM. J. Matrix Anal. and Appl., 36(2):549$579,2015$.

[14] K. Fujimoto and J. M. A. Scherpen. Balanced realization and model order reduction for nonlinear systems based on singular value analysis. SIAM J. Cont. Optim., 48(7):4591-4623, 2010. 
[15] W. S. Gray and J. Mesko. Energy Functions and Algebraic Gramians for Bilinear Systems. Proceedings of the 4th IFAC Nonlinear Control Systems Design Symposium, 31(17):101-106, 1998.

[16] W. S. Gray and J. M. A. Scherpen. On the nonuniqueness of singular value functions and balanced nonlinear realizations. Systems Control Lett., 44(3):219-232, 2001.

[17] C. Hartmann, B. Schäfer-Bung, and A. Thöns-Zueva. Balanced averaging of bilinear systems with applications to stochastic control. SIAM J. Control Optim., 51(3):2356-2378, 2013.

[18] R. Khasminskii. Stochastic stability of differential equations. Monographs and Textbooks on Mechanics of Solids and Fluids. Mechanics: Analysis, 7. Alphen aan den Rijn, The Netherlands; Rockville, Maryland, USA: Sijthoff \& Noordhoff., 1980.

[19] R. R. Mohler. Bilinear Control Processes. Academic Press, New York, 1973.

[20] B. C. Moore. Principal component analysis in linear systems: Controllability, observability, and model reduction. IEEE Trans. Autom. Control, 26:17-32, 1981.

[21] M. Redmann. Type II singular perturbation approximation for linear systems with Lévy noise. Technical report, WIAS Preprint No. 2398, 2017.

[22] M. Redmann and P. Benner. An $\mathrm{H}_{2}$-Type Error Bound for Balancing-Related Model Order Reduction of Linear Systems with Lévy Noise. Systems $\&$ Control Letters, 105:1-5, 2017.

[23] M. Redmann and P. Benner. Singular Perturbation Approximation for Linear Systems with Lévy Noise. Stochastics and Dynamics, Accepted July 2017.

[24] W. J. Rugh. Nonlinear System Theory. The Johns Hopkins University Press, Baltimore, MD, 1981.

[25] J. M. A. Scherpen. Balancing for nonlinear systems. Systems Control Lett., 21:143$153,1993$.

[26] W. H. A. Schilders, H. A. van der Vorst, and J. Rommes. Model Order Reduction: Theory, Research Aspects and Applications. Springer-Verlag, Berlin, 2008. 